\title{
Descripción de los ácidos grasos del huevo de gallinas Araucanas y Marans
}

\section{Description of the fatty acids of the eggs of Araucanas and Marans hens}

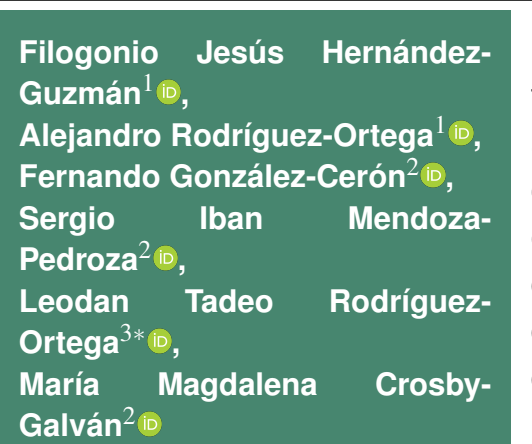

${ }^{1}$ 1Universidad Politécnica de Francisco I. Madero. Domicilio conocido s/n, CP. 42660 . Tepatepec, Hidalgo, México.

${ }^{2}$ Colegio de Postgraduados, Campus Montecillo. Carretera México-Texcoco Km. 36.5, CP. 56230. Montecillo, Texcoco, Estado de México, México.

*Autor de correspondencia: leodan.rodriguez@upfim.edu.mx

Nota científica

Recibida: 03 de marzo 2021

Aceptada: 15 de septiembre 2021

Como citar: Hernández-

Guzmán FJ, Rodríguez-Ortega A, González-Cerón F, MendozaPedroza SI, Rodríguez-Ortega LT, Crosby-Galván MM (2021) Descripción de los ácidos grasos del huevo de gallinas araucanas y marans. Ecosistemas y Recursos Agropecuarios Núm. Esp. II: e2948. DOI: 10.19136/era.a8nll.2948
RESUMEN. El perfil de ácidos grasos del del huevo es un aspecto importante en la salud del consumidor. El objetivo de este trabajo fue describir los ácidos grasos presentes en el huevo azul de gallinas Araucanas y café de gallinas Marans. El huevo azul fue similar en peso, largo y ancho que el de color café. El huevo café mostró ligeramente mayor contenido de proteína que el huevo azul, mientras que, el huevo azul tuvo mayor concentración de extracto etéreo que el huevo café. El contenido de ácido oleico y ácido docosahexaenoico fue superior en el huevo café, sin embargo, el azul presentó mayor contenido de ácido linoleico y linolénico con respecto al huevo café. En conclusión, el huevo azul de gallinas Araucanas y el huevo café de gallinas Marans contiene ácidos grasos, que de acuerdo con la literatura consultada son benéficos para la salud humana.

Palabras clave: Color del cascarón, esenciales, lípidos, salud humana.

ABSTRACT. The fatty acid profile of the egg is an important aspect of consumer health. The objective of this work was to describe the fatty acids present in the blue egg of Araucanas and brown of Marans hens. The blue egg was similar in weight, length and width to the brown one. The brown egg showed slightly higher protein content than the blue egg, while the blue egg had higher ethereal extract than the brown egg. The content of oleic acid and docosahexaenoic acid was higher in the brown egg, however, the blue one had a higher content of linoleic and linolenic acid with respect to the brown egg. In conclusion, the blue egg of Araucanas hens and the brown egg of Marans hens contain fatty acids, which according to the consulted literature, are beneficial for human health.

Key words: Shell color, essential, lipids, human health. 


\section{INTRODUCCIÓN}

El color del cascarón del huevo es una característica importante para el consumidor (Joseph et al. 1999), actualmente existe una gran diversidad de colores, desde blanco, café, verde y azul (Liu y Teng-Kuei 2010). El color depende en gran medida de la raza de las aves (Kennedy y Vevers 1976). Las gallinas Araucanas (Gallus inauris Castelló) originarias de Chile son un valioso recurso genético, debido a la producción de huevo con cascarón azul, color generado por la deposición de biliverdina en la glándula del cascarón (Zhao et al. 2006), mientras que la protoporfirina IX se ha identificado como el pigmento responsable del color café en el cascarón (Samiullah y Roberts 2013); en tanto que las gallinas Marans producen huevo con cascarón café oscuro. Al respecto, se sabe que el color del cascarón es un factor importante en la industria avícola y en la demanda del consumidor, sin embargo, el contenido nutricional del huevo es un aspecto relevante en la salud del consumidor.

El huevo contiene ácidos grasos esenciales benéficos para la salud humana, se ha observado que los ácidos grasos conocido como omegas $(\omega)$ : el oleico ( $\omega-9 ; 18$ :), linoleico $(\omega-6 ; 18: 2)$ y linolénico $(\omega-3 ; 18: 3)$ tienen efectos anticancerígenos, antiinflamatorios (Glick y Fischer 2013) y forman parte del desarrollo cerebral (Binienda et al. 2013). Además, el huevo contiene proteínas con propiedades bactericidas como lisozima (Lesnierowski et al. 2009) y ovotransferrina (Aparicio et al. 2018); pero también, también contiene colesterol (200-300 mg por $100 \mathrm{~g}$ ) como lo señala Miranda et al. (2015), lo que ha hecho que disminuya su consumo. Sin embargo, el colesterol tiene funciones importantes en el cuerpo humano, es generador de hormonas como: testosterona y estradiol, y es precursor de vitamina $\mathrm{D}$ (Mattila et al. 2003) y sales biliares útiles para digerir y absorber las grasas (Priya et al. 2013). El contendido de ácidos grasos del huevo se ve afectado por la genética de las aves (Stadelman y Pratt 1989). Debido a que en la revisión de literatura realizada se encontraron pocos informes sobre el perfil de ácidos grasos del huevo azul de gallinas Araucanas y café de gallinas Marans. El objetivo de este trabajo fue describir el perfil de ácidos grasos presentes en el huevo azul de gallinas Araucanas (Gallus inauris Castelló) y del huevo café de gallinas Marans (Gallus gallus domesticus).

\section{MATERIALES Y MÉTODOS}

El trabajo se desarrolló en la unidad avícola de la Universidad Politécnica de Francisco I. Madero, ubicada a $1900 \mathrm{~m}$ de altitud (Rodríguez-Ortega et al. 2020) en Tepatepec, Hidalgo México. Se utilizaron 10 gallinas Araucanas con peso vivo promedio (PV) de $1.775 \pm 0.250 \mathrm{~kg}$ y 10 gallinas Marans (PV: 2.279 $\pm 0.119 \mathrm{~kg}$ ), previamente colectadas de la avicultura de traspatio que se desarrolla en el Valle del Mezquital Hidalgo, México (Figura 1). Los animales fueron alimentados ad libitum con alimento comercial peletizado (proteína cruda $17 \%$, grasa $3 \%$, cenizas $15 \%$, humedad $12 \%$, fibra $11 \%$, extracto libre de nitrógeno $42 \%$ ) que cubrió sus necesidades de mantenimiento (NRC 1994), el agua se ofreció a libre acceso. Los animales fueron alojados en corrales de $2 \times 4 \mathrm{~m}$, techados y con piso de tierra. El huevo se colectó todos los días y se registró el peso con una báscula digital de $200 \mathrm{~g}$ con resolución de $0.01 \mathrm{~g}$ (Modelo MH-200, Marca MKS Tools), mientras que el largo y ancho se determinaron con un vernier digital (Modelo HER-411, STEREN) con rango de medición de 0 a $150 \mathrm{~mm}$ y resolución de $0.1 \mathrm{~mm}$, la colecta de de huevo se realizó durante cinco semanas hasta reunir 100 huevos de ambas razas de gallinas.

\section{Análisis del huevo}

El huevo se colectó y almacenó a temperatura ambiente en un lugar obscuro hasta su liofilización (Liofilizadora Labconco), debido a que este método de deshidratación no altera la estructura química del material (Surco-Laos et al. 2017). La determinación de cenizas se desarrolló de acuerdo al método descrito por Cuello et al. (2017). El contenido de grasa se determinó usando el método de extracción Soxhlet. Para lo cual a partir de la pérdida de peso después de la extracción de lípidos con éter dietílico en el aparato Soxhlet se calculó el contenido de grasa 

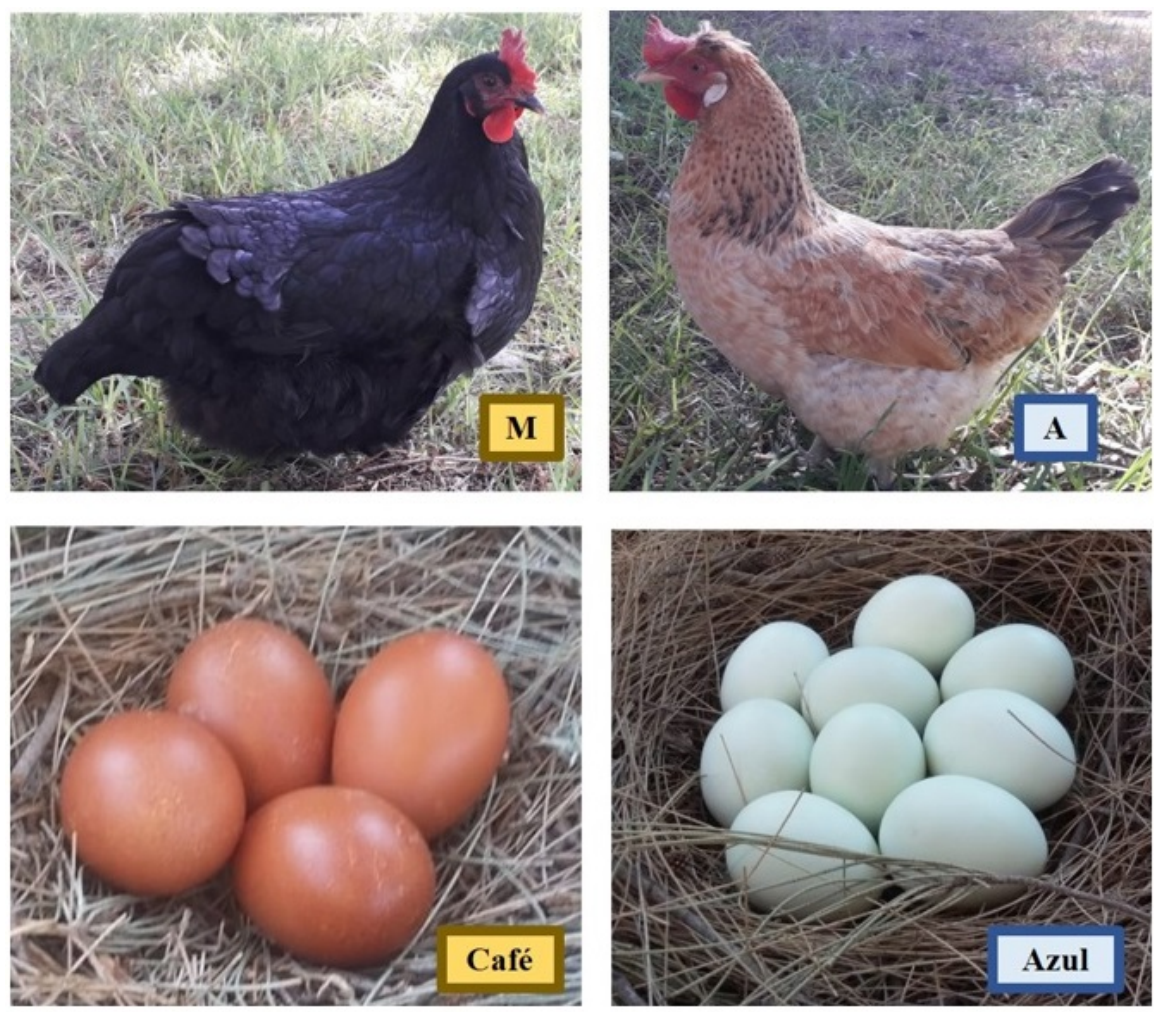

Figura 1. Gallinas Marans (M), huevo café obscuro (café), gallinas Araucanas (A), huevo azul (azul).

(González et al. 2009). Mientras que, el contenido de proteína cruda se determinó mediante el método de Kjeldahl (Sun et al. 2019). El perfil de ácidos grasos del huevo azul y café se determinó con la metodología de Palmquist y Jenkins (2003) y Jenkins (2010), en un cromatógrafo de gases HP 6890 con inyector automático y detector de ionización de flama con columna capilar SUPELCO SP 2560 de $100 \mathrm{~m}$, diámetro interno de $0.25 \mathrm{~mm}$ y 0.2 micras de película, con helio como gas acarreador. Para la identificación de los ácidos grasos se utilizó un estándar FAME Mix C4 -C24 100 mg SUPELCO con número de catálogo 18919-1 en ampolleta (St. Louis, MO, USA).

\section{Análisis estadístico}

El peso, largo, ancho del huevo se analizaron con el procedimiento PROC UNIVARIATE y con el procedimiento PROC TEST (SAS 2011), mientras que la determinación de ácidos grasos se realizó en una sola repetición debido a los elevados costos del análisis, por lo que solo se describen los resultados.

\section{RESULTADOS Y DISCUSIÓN}

El huevo azul de gallinas Araucanas fue similar $(p>0.05)$ en peso, largo y ancho que el huevo café de gallinas Marans (Tabla 1), el huevo producido por ambas razas no se considera de pequeñas dimensiones, ya que las aves criollas de traspatio producen huevo de menor tamaño. Al respecto, Juárez-Caratachea et al. (2010) reportan que el peso de huevo de las gallinas criollas es de $50.7 \mathrm{~g}$, mientras que, el diámetro longitudinal y transversal se encuentra entre 5.6 y $4.2 \mathrm{~cm}$. El huevo azul presentó mayor contenido de materia seca (MS) y cenizas que el huevo café, pero el huevo café tuvo cinco unidades porcentuales de proteína más que el huevo azul, mientras que, el huevo azul mostró mayor concentración de extracto etéreo que el huevo café (Tabla 2). Tanto el huevo azul como el de color café tuvieron un contenido de cenizas similar al huevo comercial (3.79 y 3.62 vs $3.92 \%$, Abdul et al. 2016). Pero para el contenido de extracto etéreo fue mayor en el huevo azul y café 
que en el huevo de gallinas Naked Neck ó de cuello desnudo de 31.69 y 24.83 vs $11.39 \%$ (Stanišic et al. 2015), por lo que debido al elevado contenido de extracto etéreo se sugiere consumirse con moderación.

Tabla 1. Peso, ancho y largo de huevo azul de gallinas Araucanas y café de gallinas Marans.

\begin{tabular}{lccc}
\hline Huevo & Peso $(\mathrm{g})$ & Ancho $(\mathrm{cm})$ & Largo $(\mathrm{cm})$ \\
\hline Azul & $62.16^{a}$ & $4.33^{a}$ & $5.73^{a}$ \\
Café & $62.58^{a}$ & $4.30^{a}$ & $5.85^{a}$ \\
Error Estándar & 0.63 & 0.16 & 0.26 \\
\hline \multicolumn{7}{l}{ Medias con diferente letra, dentro de cada columna, son } \\
estadísticamente diferentes $(\mathrm{p}<0.05)$.
\end{tabular}

Tabla 2. Descripción del contenido nutricional del huevo azul de gallinas Araucanas y café de gallinas Marans en base seca.

\begin{tabular}{lcccc}
\hline Huevo & MS (\%) & Cenizas (\%) & Proteína (\%) & Extracto etéreo \\
\hline Azul & 76.15 & 3.79 & 38.65 & 31.69 \\
Café & 72.27 & 3.62 & 43.81 & 24.83 \\
\hline
\end{tabular}

El huevo café de gallinas Marans presentó similar contenido de ácido palmítico que el huevo azul de gallinas Araucanas (Tabla 3 ) y que el huevo comercial (27.72 y 26.48 vs $27.29 \%$, Sujiwo et al. 2017). El consumo elevado de ácido palmítico puede incrementar los niveles de colesterol y el tejido adiposo en el cuerpo humano (Carta et al. 2017), por lo que es recomendable consumir bajos niveles de este ácido graso. Pero en contraparte este ácido graso es crucial para la síntesis de ácido palmitoleico, pero este último no se considera esencial (Coronado et al. 2006). El ácido palmitoleico fue ligeramente superior en el huevo café con respecto al huevo azul, pero huevo café y el azul tuvieron mayor contenido de ácido palmitoleico (Tabla 3) que el huevo comercial (3.55 y 2.40 vs $2.02 \%$, Sujiwo et al. 2017).

El huevo comercial tiene ligeramente un mayor contenido de ácido esteárico (12.39\%, Sujiwo et al. 2017) que el huevo azul y café (9.83 y $9.36 \%)$. Al respecto, se ha reportado que el ácido esteárico tiene efectos beneficiosos en la salud humana, por ejemplo, Mensink (2005) reporta que el consumo de ácido esteárico reduce ligeramente el colesterol. El huevo de gallinas Araucanas y Marans contiene bajo porcentaje de ácido araquidónico en comparación con el huevo comercial $(1.79,2.15$ vs $4.32 \%$, Sujiwo et al. 2017), el cual funciona como precursor de las prostaglandinas, prostaciclinas y tromboxanos (Cantú et al. 2017), por lo que es benéfico para la salud humana.

Tabla 3. Contenido de ácidos grasos del huevo azul de gallinas Araucanas y café de gallinas Marans.

\begin{tabular}{llcc}
\hline & & \multicolumn{2}{c}{$\%$} \\
\cline { 3 - 4 } Ácido graso & Nombre del ácido graso & Araucana & Marans \\
\hline C14:0 & Mirístico & 0.39 & 0.37 \\
C15:0 & Pentadecanoico & 0.07 & 0.07 \\
C16:0 & Palmítico & 26.48 & 27.72 \\
C16:1n7 & Palmitoleico (Omega 7) & 2.40 & 3.55 \\
C17:0 & Heptadecanoico & 0.22 & 0.21 \\
C18:0 & Esteárico & 9.83 & 9.36 \\
C18:1n9 & Oleico (Omega 9) & 37.51 & 39.40 \\
C18:2n6 & Linoleico (Omega 6) & 16.52 & 13.85 \\
C18:3n3 & Linolénico (Omega 3) & 0.44 & 0.39 \\
C20:0 & Araquidónico & 1.79 & 2.15 \\
C22:6n3 & Docosahexaenoico (DHA) & 0.47 & 0.58 \\
Desconocida & Otros ácidos grasos & 3.88 & 2.35 \\
\hline
\end{tabular}

El ácido mirístico (C14:0) fue ligeramente superior en el huevo azul y en el de color café en comparación con el huevo comercial (0.39 y 0.37 vs $0.29 \%$, Sujiwo et al. 2017). Este ácido fue encontrado por primera vez en la nuez moscada (Myristica fragrans) de donde procede su nombre. El ácido mirístico es fundamental en recién nacidos, debido a que estimula su apetito, por otra parte, es fuente de grupos miristoilo utilizados dentro del cuerpo para estabilizar proteínas en el sistema inmune (Uno et al. 2004), característica importante en la salud humana, lo que evidencia la importancia del consumo de huevo de estas gallinas. Las porciones de ácido linoleico y linolénico fueron ligeramente superiores en el huevo azul de gallinas Araucanas en comparación con el huevo café de gallinas Marans (Tabla 3). Al comparar el huevo de Araucanas con el huevo comercial, el ácido linoleico (16.52 vs $12.37 \%$ ) y linolénico (0.44 vs $0.20 \%$ ) se encontraron en mayor proporción, respectivamente. Estos ácidos grasos insaturados que pertenece a la familia de los omegas $(\omega)$ : $\omega$-3 (ácido linolénico) y $\omega$-6 (ácido linoleico) son considerados esenciales, debido a que el ser humano no cuenta con las enzimas necesarias para sintetizarlos (Valenzuela et al. 2011). Por lo que son el foco de interés para los consumidores debido sus efectos anticancerígenos, antioxidantes y neurológi- 
cos. El huevo de Araucana presento $0.24 \%$ mayor contenido de ácido linolénico con respecto al huevo comercial, lo cual es benéfico para la salud humana, ya que puede transformar vía desaturasas y elongasas en los ácidos eicosapentaenoico (C20:5, EPA) y docosahexaenoico (C22:6 n3, DHA) que son necesarios para el desarrollo neurológico (Molina-Peralta y Mach 2014).

El huevo café mostró mayor contenido de ácido oleico (omega 9) que el huevo azul (Tabla 3) y que el huevo comercial (39.40 vs 37.51, 35.77\%; Sujiwo et al. 2017). Al respecto, Tin (2005) reportó que el consumo de ácido oleico reduce el crecimiento de células cancerígenas, debido a que inhibe la actividad de la enzima ciclooxigenasa (COX-2). Por lo que se observa que el huevo de color café y de color azul es un alimento que no afecta de formam negativa la salud humana, además de que se ha reportado que el huevo azul contiene $\alpha$ - glucosidasa, enzima esencial para el catabolismo de glucógeno a glucosa en los lisosomas. Lo que evita la sobreacumulación de glucógeno en diferentes células del cuerpo, además de intervenir en la hidrólisis del almidón (Sujiwo et al. 2017). El contenido de ácido docosahexaenoico (DHA, C22: 6n3) fue ligeramente superior en el huevo café de gallinas Marans que en el huevo azul (Tabla 3), ácido fundamental para el desarrollo visual y neurológico, además de componente mayoritario de la estructura cerebral (Gil-Campos et al. 2010). El DHA es precursor de docosanoides (metabolitos del DHA, Valenzuela et al. 2013) de los que deriva la neuroprotectina D1 con propiedades neuroprotectoras frente al envejecimiento cerebral, debido a que inhibe el estrés oxidativo y favorece la supervivencia celular.

Los resultados evidencian que el huevo azul de gallinas Araucanas y el huevo café de gallinas Marans presentan similar peso, largo y ancho. Pero el huevo azul de gallinas Araucanas tiene mayor contenido de ácido linoleico y linolénico, mientras que el huevo de gallinas Marans prsenta mayor contenido de ácido docosahexaenoico. Pero ambos tipos de huevo contiene ácidos grasos benéficos para la salud humana.

\section{LITERATURA CITADA}

Abdul RS, Akhter S, Hassan KS, Ashraf AM (2016) A comparative study on quality, proximate composition and cholesterol content of eggs and meat in Fayoumi and commercial White Leghorn chickens. Cogent Food and Agriculture 2: 1-7. DOI: 10.1080/23311932.2016.1195539

Aparicio A, Salas GMD, Cuadrado-Soto E, Ortega RM, López-Sobaler AM (2018) El huevo como fuente de antioxidantes y componentes protectores frente a procesos crónicos. Nutrición Hospitalaria 35: 36-40.

Binienda ZK, Sarkar S, Silva-Ramirez S, Gonzalez C (2013) Role of free fatty acids in physiological conditions and mitochondrial dysfunction. Food and Nutrition Sciences 4: 6-15.

Cantú SM, Jin LH, Donoso A, Puyó AM, Peredo HA (2017) El ácido araquidónico y sus derivados. generalidades de los prostanoides en relación con procesos inflamatorios. Ciencia e Investigación 67: 5-12.

Carta G, Murru E, Banni S, Manca C (2017) Palmitic acid: physiological role, metabolism and nutritional implications. Frontiers in Physiology 8: 902. DOI: 10.3389/fphys.2017.00902.

Coronado HM, Vega LS, Gutiérrez TR, García FB, Díaz GG (2006) Los ácidos grasos omega-3 y omega-6: nutrición, bioquímica y salud. Revista de Educación Bioquímica 25: 72-79.

Cuello M, Jaramillo GK, Canchiungre E, Pérez JC, Castro C, Cabrera O (2017) Determinación de componentes nutricionales presentes en las hojas secas de Annona muricata L. (Guanábana). Revista CUMBRES 3: 9-16.

González AD, Kafarov V, Monsalve AG (2009) Desarrollo de métodos de extracción de aceite en la cadena de producción de biodiesel a partir de microalgas. Prospectiva 7: 53-60. 
Gil-Campos M, Dalmau SJ, Comité de Nutrición de la Asociación Española de Pediatría (2010) Importancia del ácido docosahexaenoico (DHA): funciones y recomendaciones para su ingesta en la infancia. Anales de Pediatria 73: 142.e1-142.e8.

Glick RN, Fischer MH (2013) The role of essential fatty acids in human health. Journal of Evidence-Based Complementary and Alternative Medicine 18: 268-289.

Jenkins TC (2010) Technical note: Common analytical errors yielding inaccurate results during Analysis of fatty acids in feed and digesta samples. Journal of Dairy Science 93: 1170-1174.

Juárez-Caratachea A, Gutiérrez-Vázquez E, Segura-Correa J, Santos-Ricalde R (2010) Calidad del huevo de gallinas criollas criadas en traspatio en Michoacán, México. Tropical and Subtropical Agroecosystems 12: 109-115.

Joseph NS, Robinson NA, Renema RA, Robinson FE (1999) Shell quality and color variation in broiler breeder egg. Journal of Applied Poultry Research 8: 70-74.

Kennedy GY, Vevers HG (1976) A survey of avian eggshell pigments. Comparative Biochemistry and Physiology 55: 117-123.

Lesnierowski G, Kijowski J, Cegielska-Radziejewska R (2009) Ultrafiltration-modified chicken egg white lysozyme and its antibacterial action. International Journal of Food Science and Technology 44: 305-311.

Liu H-C, Teng-Kuei CW (2010) Eggshell pigmentation: a review. Journal of the Chinese Society of Animal Science 39: 75-89.

Mattila P, Rokka T, Nko KK, Valaja J, Rossow L, Ryhanen EL (2003) Effect of cholecalciferol-enriched hen feed on egg quality. Journal Agricultural Food Chemistry 51: 283-287.

Mensink RP (2005) Effects of stearic acid on plasma lipid and lipoproteins in Humans. Lipids 40: 1201-1205.

Miranda JM, Anton X, Redondo-Valbuena C, Roca-Saavedra P, Rodriguez JA, Lamas A, Franco CM, Cepeda A (2015) Egg and egg-derived foods: Effects on human health and use as functional foods. Nutrients 7 : 706-729.

Molina-Peralta Á, Mach N (2014) Alimentos ricos en ácidos grasos v-3 libres de contaminantes y aptos para vegetarianos, y su importancia en el desarrollo neurológico normal. Revista Española de Nutrición Humana y Dietética 18: 89-99.

NRC (1994) Nutrient requirements of poultry. National Research Council. 9th Edition. National Academy Press, Washington, DC, USA. 176p.

Palmquist DL, Jenkins TC (2003) Challenges with fats and fatty acid methods. Journal Animal Science. 81: 250-354.

Priya T, Maurya S, Khan KH (2013) Cholesterol: Genetic, clinical and natural implications. Research Journal of Pharmaceutical Biological and Chemical Sciences 4: 1344-1364.

Rodríguez-Ortega LT, Rodríguez-Ortega A, Hernández-Guzmán FJ, Callejas-Hernández J, Pro-Martínez A, LeyvaJimenez H (2020) Productive performance and egg physical characteristics of Tufted Creole and Marans hens. Agroproductividad 13: 69-73.

Samiullah S, Roberts JR (2013) The location of protoporphyrin in the eggshell of brown-shelled eggs. Poultry Science 92: 2783-2788.

Stadelman WJ, Pratt DE (1989) Factors influencing composition of the hen's egg. World's Poultry Science 45: 247-266. 
Sujiwo J, Kim D, Yoon J-Y, Kim H, Kim J-S, Lee S-K, Jang A (2017) Physicochemical and functional characterization of blue-shelled eggs in korea. Korean Journal Food Science of Animal 37: 181-190.

Sun C, Liu J, Yang N, Xu G (2019) Egg quality and egg albumen property of domestic chiken, duck, goose, turkey, and pigeon. Poultry Science 0: 1-6.

Surco-Laos F, Tipiana R, Torres Y, Valle M, Panay J (2017) Efectos de liofilización sobre composición química y capacidad antioxidante en pulpa de cuatro variedades de Mangifera indica. Revista de la Sociedad Química del Perú 83: 412-419.

Stanišic N, Petricevic V, Škrbic Z, Lukic M, Pavlovski Z, Lilic S, Petricevic M (2015) Effects of age and time of day of sampling on proximate and fatty acid composition of whole eggs from two strains of laying hens. Archives Animal Breeding 58: 151-158.

Tin WD (2005) Oleic acid - the anti-breast cancer component in olive oil. Australian Journal Technology 9: 75-78.

Uno F, Sasaki J, Nishizaki M, Carboni G, Xu K, Atkinson NE, Kondo M, Minna DJ, Roth AJ, Ji L (2004) Myristoylation of the fus 1 protein is required for tumor suppression in human lung cancer cells. Cancer Research 64: 2969-2976.

Valenzuela BR, Tapia OG, González EM, Valenzuela BA (2011) Ácidos grasos omega-3 (EPA y DHA) y su aplicación en diversas situaciones clínicas. Revista Chilena de Nutrición 38: 356-367.

Valenzuela BR, Morales PJ, Sanhueza CJ, Valenzuela BA (2013) Ácido docosahexaenoico (DHA), un ácido graso esencial a nivel cerebral. Revista Chilena de Nutrición 40: 383-390.

Zhao R, Xu G-Y, Liu Z-Z, Li J-Y, Yang N (2006) A Study on Eggshell Pigmentation: Biliverdin in Blue-Shelled Chickens. Poultry Science 85: 546-549. 